\title{
Bound State Solutions of the Hua Potential within the Framework of Two Approximations Scheme
}

\author{
E. B. Ettah, P. O. Ushie, and C. M. Ekpo
}

\section{ABSTRACT}

In this paper, we solve analytically the Schrodinger equation for s-wave and arbitrary angular momenta with the Hua potential is investigated respectively. The wave function as well as energy equation are obtained in an exact analytical manner via the Nikiforov Uvarov method using two approximations scheme. Some special cases of this potentials are also studied.

Keywords: Schrodinger equation; Exact solution; Hua potential.

\author{
Published Online: June 24, 2021 \\ ISSN: $2684-4451$ \\ DOI : 10.24018/ejphysics.2021.3.3.72

\section{E. B. Ettah} \\ Department of Physics, Cross River \\ University of Technology, Calabar, \\ Nigeria. \\ (e-mail: emmanuel.ettah@gmail.com) \\ P. O- Ushie* \\ Department of Physics, Cross River \\ University of Technology, Calabar, \\ Nigeria. \\ (e-mail: patushie98@gmail.com) \\ C. M. Ekpo \\ Department of Physics, Cross River \\ University of Technology, Calabar, \\ Nigeria. \\ (e-mail: chrisekpo25@gmail.com) \\ *Corresponding Author
}

\section{INTRODUCTION}

It is well known that Bound state solutions of Schrodinger equation play an integral role in Quantum Mechanics. Bound state solutions describe the system of a particle subjected to a potential, with the tendency to remain in a fixed region of space [1]. Quantum mechanical Wave functions and their corresponding eigenvalues give significant information in describing various quantum systems [2]-[21].

Ikot et al. [20] studied the Dirac equation in the presence of the modified Mobius square potential with a Yukawa-like tensor interaction and obtain the eigenvalues and corresponding eigenfunctions for any -state by using Nikiforov-Uvarov method [20]. Ekpo et al [22] solved the Schrodinger equation for the New Generalized Morse-Like Potential in arbitrary dimensions by using the Nikiforov Uvarov Method. Hua potential [18], proposed by Hua as an intermolecular potential and widely applied to molecular physics and quantum chemistry.

The Hua potential is given [18]:

$V(r)=V_{1}\left(\frac{1-e^{-2 \alpha r}}{1-q e^{-2 \alpha r}}\right)^{2}$

It has been established that the solution for the Schrodinger equation for $l \neq 0$, one has to use a Pekeris-type approximation scheme to deal with the centrifugal term or solve numerically. The following new improved approximation scheme to deal with the centrifugal term (Pekeris-type approximation scheme) [14]-[17]:

$$
\begin{aligned}
& \frac{1}{r^{2}}=4 \alpha^{2}\left(C_{0}+\frac{e^{-2 \alpha r}}{1-e^{-2 \alpha r}}\right)=4 \alpha^{2}\left(C_{0}+\frac{e^{-2 \alpha r}}{1-e^{-2 \alpha r}}+\right. \\
& \left.\left(\frac{e^{-2 \alpha r}}{1-e^{-2 \alpha r}}\right)^{2}\right)
\end{aligned}
$$

Greene-Aldrich approximation scheme [11], which is given by:

$\frac{1}{r^{2}}=\frac{4 \alpha^{2} e^{-2 \alpha r}}{\left(1-e^{-2 \alpha r}\right)^{2}}$

This paper is organized as follows. In Sect. 2, the review of the NU method is presented. In Sect. 3, this method is applied to the radial Schrodinger equation to find the analytical solution with Hua potential. In Sect. 3, the NU method is also applied to radial Schrodinger equation with Hua potential. In Sect. 4, a brief conclusion is given.

\section{REVIEW OF NIKIFOROV-UVAROV METHOD}

The Nikiforov-Uvarov (NU) method is based on solving the hypergeometric-type second-order differential equations by means of the special orthogonal functions [18]. The main equation which is closely associated with the method is given in the following form [19]. 
$\psi^{\prime \prime}(s)+\frac{\tilde{\tau}(s)}{\sigma(s)} \psi^{\prime}(s)+\frac{\widetilde{\sigma}(s)}{\sigma^{2}(s)} \psi(s)=0$

where $\sigma(s)$ and $\tilde{\sigma}(s)$ are polynomials at most second-degree, $\tilde{\tau}(s)$ is a first-degree polynomial and $\psi(s)$ is a function of the hypergeometric-type.

The exact solution of (2) can be obtained by using the transformation:

$\psi(s)=\phi(s) y(s)$

This transformation reduces (2) into a hypergeometric-type equation of the form:

$\sigma(s) y^{\prime \prime}(s)+\tau(s) y^{\prime}(s)+\lambda y(s)=0$

The function $\phi(s)$ can be defined as the logarithm derivative:

$\frac{\phi^{\prime}(s)}{\phi(s)}=\frac{\pi(s)}{\sigma(s)}$

where $\pi(s)=\frac{1}{2}[\tau(s)-\tilde{\tau}(s)]$

with $\pi(s)$ being at most a first-degree polynomial. The second $\psi(s)$ being $y_{n}(n)$ in (3), is the hypergeometric function with its polynomial solution given by Rodrigues relation:

$y^{(n)}(s)=\frac{B_{n}}{\rho(s)} \frac{d^{n}}{d s^{n}}\left[\sigma^{n} \rho(s)\right]$

Here, $B_{n}$ is the normalization constant and $\rho(s)$ is the weight function which must satisfy the condition:

$(\sigma(s) \rho(s))^{\prime}=\sigma(s) \tau(s)$

$\tau(s)=\tilde{\tau}(s)+2 \pi(s)$

It should be noted that the derivative of $\tau(s)$ with respect to $s$ should be negative. The wavefunction and energy equation can be obtained using the definition of the following function $\pi(s)$ and parameter $\lambda$, respectively:

$\pi(s)=\frac{\sigma^{\prime}(s)-\tilde{\tau}(s)}{2} \pm \sqrt{\left(\frac{\sigma^{\prime}(s)-\tilde{\tau}(s)}{2}\right)^{2}-\tilde{\sigma}(s)+k \sigma(s)}$

where $k=\lambda-\pi^{\prime}(s)$

The value of $k$ can be obtained by setting the discriminant of the square root in (9) equal to zero. As such, the new eigenvalue equation can be given as:

$\lambda_{n}=-n \tau^{\prime}(s)-\frac{n(n-1)}{2} \sigma^{\prime \prime}(s), n=0,1,2, \ldots$

\section{Bound State Solution with Hua Potential}

The time independent Schrodinger equation can be given as: $\frac{d^{2} R_{n l}}{d r^{2}}+\frac{2 \mu}{\hbar^{2}}\left[E_{n l}-V(r)-\frac{\hbar^{2} \ell(\ell+1)}{2 \mu r^{2}}\right] R_{n l}=0$

where $\mu$ is the reduced mass, $E_{n l}$ is the energy spectrum, $\hbar$ is the reduced Planck's constant and $n$ and $l$ are the radial and orbital angular momentum quantum numbers respectively (or vibration-rotation quantum number in quantum chemistry).

Again, substituting (1a) into (12) gives:

$\frac{d^{2} R_{n l}}{d r^{2}}+\frac{2 \mu}{\hbar^{2}}\left[E_{n l}-V_{1}\left(\frac{1-e^{-2 \alpha r}}{1-q e^{-2 \alpha r}}\right)^{2}-\frac{\hbar^{2} \ell(\ell+1)}{2 \mu r^{2}}\right] R_{n l}=0$

Substituting Approximation 2 (1e) and $q$ deforming .(1c), (13) becomes:

$\frac{d^{2} R_{n l}}{d r^{2}}+\frac{2 \mu}{\hbar^{2}}\left[E_{n l}-V_{1}\left(\frac{1-e^{-2 \alpha r}}{1-q e^{-2 \alpha r}}\right)-\right.$
$\left.\frac{\hbar^{2} \ell(\ell+1)}{2 \mu}\left(\frac{4 \alpha^{2} e^{-2 \alpha r}}{\left(1-q e^{-2 \alpha r}\right)^{2}}\right)\right] R_{n l}=0$

Rearranging (14) we get:

$\frac{d^{2} R_{n \ell}(r)}{d r^{2}}+\frac{2 \mu}{\hbar^{2}\left(1-q e^{-2 \alpha r}\right)^{2}}\left[E_{n l}\left(1-q e^{-2 \alpha r}\right)^{2}-V_{1}(1-\right.$
$\left.\left.e^{-2 \alpha r}\right)^{2}-\frac{2 \alpha^{2} e^{-2 \alpha \hbar^{2} \ell(\ell+1)}}{\mu}\right] R_{n \ell}(r)=0$

Again, invoking the transformation $s=e^{-\alpha r}$ in (15) we get:

$\frac{d^{2} R_{n \ell}(s)}{d s^{2}}+\frac{1-q s}{s(1-q s)} \frac{d R_{n \ell}(s)}{d s}+\frac{1}{s^{2}(1-q s)^{2}}\left[-s^{2}\left(\varepsilon_{n} q^{2}+\beta\right)+\right.$

$\left.s\left(2 \varepsilon_{n} q+2 \beta-\gamma\right)-\left(\varepsilon_{n}+\beta\right)\right] R_{n \ell}(s)=0$

where

$\left\{-\varepsilon_{n}=\frac{\mu E_{n \ell}}{2 \hbar^{2} \alpha^{2}} \beta=\frac{\mu V_{1}}{2 \hbar^{2} \alpha^{2}}\right.$ and $\left.\gamma=\ell(\ell+1)\right\}$

Comparing (16) and (2), we have the following parameters:

$\left\{\begin{array}{c}\tilde{\tau}(s)=1-q s \\ \sigma(s)=s(1-q s) \\ \tilde{\sigma}(s)=-s^{2}\left(\varepsilon_{n} q^{2}+\beta\right)+ \\ s\left(2 \varepsilon_{n} q+2 \beta-\gamma\right)-\left(\varepsilon_{n}+\beta\right)\end{array}\right\}$

Substituting these polynomials into (9), we get $\pi(s)$ to be:

$\pi(s)=-\frac{q s}{2} \pm \sqrt{\begin{array}{c}\left(\frac{q^{2}}{4}+\varepsilon_{n} q^{2}+\beta-k q\right) s^{2}+ \\ \left(-2 \varepsilon_{n} q-2 \beta+\gamma+k\right) s+\varepsilon_{n}+\beta\end{array}}$

To find the constant $k$, the discriminant of the expression under the square root of (19) must be equal to zero. As such, we have that:

$k \pm=(2 \beta-\gamma-2 \beta q) \pm$

$2 \sqrt{\varepsilon_{n}+\beta} \sqrt{\left(\frac{q^{2}}{4}-2 \beta q+\beta q^{2}+\beta+\gamma q\right)}$

Substituting (20) into (19) yields: 
$\pi(s)=-\frac{q s}{2} \pm\left[\left(\sqrt{\frac{q^{2}}{4}-2 \beta q+\beta q^{2}+\beta+\gamma q}+\right.\right.$

$\left.\left.q \sqrt{\varepsilon_{n}+\beta}\right) s-\sqrt{\varepsilon_{n}+\beta}\right]$

Again, from the knowledge of NU method, we choose the expression $\pi(s)_{-}$which the function $\tau(s)$ has a negative derivative. This is given by:

$k_{-}=(2 \beta-\gamma-2 \beta q)-$

$2 \sqrt{\varepsilon_{n}+\beta} \sqrt{\left(\frac{q^{2}}{4}-2 \beta q+\beta q^{2}+\beta+\gamma q\right)}$

with $\tau(s)$ being obtained as:

$\tau(s)=1-2 q s-2\left[\left(\sqrt{\frac{q^{2}}{4}-2 \beta q+\beta q^{2}+\beta+\gamma q}+\right.\right.$

$\left.\left.q \sqrt{\left(\varepsilon_{n}+\beta\right)}\right) s-\sqrt{\left(\varepsilon_{n}+\beta\right)}\right]$

Referring to (10), we define the constant $\lambda$ as:

$\lambda=(2 \beta-\gamma-2 \beta q)-$

$2 \sqrt{\varepsilon_{n}+\beta} \sqrt{\left(\frac{q^{2}}{4}-2 \beta q+\beta q^{2}+\beta+\gamma q\right)}-$

$\frac{q}{2}\left[\left(\sqrt{\frac{q^{2}}{4}-2 \beta q+\beta q^{2}+\beta+\gamma q}+q \sqrt{\varepsilon_{n}+\beta}\right)\right]$

Substituting (24) into (11) and carrying out simple algebra, where:

$\tau^{\prime}(s)=-2 q-2\left(\sqrt{\left(\frac{1}{4}-\delta+\gamma-\eta-\beta\right)}+\right.$

$\left.q \sqrt{\left(\varepsilon_{n}+\beta\right)}\right)<0$

And

$\sigma^{\prime \prime}(s)=-2 q$

We have:

$\varepsilon_{n}=-\beta+\frac{1}{4}\left[\frac{\beta\left(\frac{1}{q^{2}}-1\right)-\left(n+\frac{1}{2}+\sqrt{\frac{1}{4}-\frac{2 \beta}{q}+\beta+\frac{\beta}{q^{2}}-\frac{\gamma}{q}}\right)^{2}}{\left(n+\frac{1}{2}+\sqrt{\frac{1}{4}-\frac{2 \beta}{q}+\beta+\frac{\beta}{q^{2}}-\frac{\gamma}{q}}\right)}\right]^{2}$

Substituting (17) into (27) gives the energy eigenvalue equation of the Hua potential in the form:

$E_{n \ell}{ }^{(\text {Approx } .2)}=V_{1}-\frac{\hbar^{2} \alpha^{2}}{2 \mu}\left[\frac{\frac{\mu V_{1}}{2 \hbar^{2} \alpha^{2}}\left(\frac{1}{q^{2}}-1\right)-\zeta^{2}}{\zeta}\right]^{2}$

The corresponding wave functions can be evaluated by substituting $\pi(s)_{-}$and $\sigma(s)$ from (18) and (21), respectively into (5) and solving the first order differential equation. This gives:
$\Phi(s)=s^{\sqrt{\varepsilon_{n}+\beta}}(1-q)^{\frac{1}{2}+\sqrt{\frac{1}{4}-\frac{2 \beta}{q}+\beta+\frac{\beta}{q^{2}}+\frac{\gamma}{q}}}$

The weight function $\rho(s)$ from (7) can be obtained as:

$\rho(s)=s^{2 \sqrt{\varepsilon_{n}+\beta}}(1-q s)^{2 \sqrt{\frac{1}{4}-\frac{2 \beta}{q}+\beta+\frac{\beta}{q^{2}}+\frac{\gamma}{q}}}$

From the Rodrigues relation of (6), we obtain:

$y_{n}(s) \equiv N_{n, l} P_{n}^{\left(2 \sqrt{\varepsilon_{n}+\beta}, 2 \sqrt{\frac{1}{4}-\frac{2 \beta}{q}+\beta+\frac{\beta}{q^{2}}+\frac{\gamma}{q}}\right)}(1-2 q s)$

where $P_{n}^{(\theta, \vartheta)}$ is the Jacobi Polynomial.

Substituting $\Phi(s)$ and $y_{n}(s)$ from (29) and (31) respectively into (3), we obtain the unnormalized wave function as:

$R_{n}(s)=N_{n, l} S^{\sqrt{\varepsilon_{n}+\beta}}(1-$
$q s)^{\frac{1}{2}+\sqrt{\frac{1}{4}-\frac{2 \beta}{q}+\beta+\frac{\beta}{q^{2}}+\frac{\gamma}{q}}} P_{n}^{\left(2 \sqrt{\varepsilon_{n}+\beta}, 2 \sqrt{\frac{1}{4}-\frac{2 \beta}{q}+\beta+\frac{\beta}{q^{2}}+\frac{\gamma}{q}}\right)}(1-2 q s)(32$

Again, by using approximation 1 (1b) and repeating the above procedure, we can consequently obtain the energy eigenvalues as:

$$
\begin{gathered}
E_{n \ell}{ }^{(\text {Approx.1) }}=V_{1}+\frac{2 \hbar^{2} \alpha^{2} C_{0} \ell(\ell+1)}{\mu}- \\
\frac{\hbar^{2} \alpha^{2}}{2 \mu}\left[\frac{\frac{\mu V_{1}}{2 \hbar^{2} \alpha^{2}}\left(\frac{1}{q^{2}}-1\right)-\zeta^{2}}{\zeta}\right]^{2} \\
\zeta=\left(n+\frac{1}{2}+\sqrt{\left.\frac{1}{4}+\frac{\mu V_{1}}{2 \hbar^{2} \alpha^{2}}\left(\frac{1}{q}-1\right)^{2}+\frac{\ell(\ell+1)}{\mathrm{q}}\right)}\right.
\end{gathered}
$$

\section{CONCLUSION}

In this work, we have investigated non-relativistic problem of Schrodinger equation subject to the Hua potential respectively within the framework of two approximations scheme. We have obtained exact energy eigenvalues equation and radial wave functions using the NU method. Finally, we note that our result agrees with existing literature. The energy equation and wave function can be used to extend the study to information theory, effects of external fields (magnetic and Aharanov-Bohm (AB) fields), scattering state and thermodynamic properties [26]-[33].

\section{REFERENCES}

[1] A.N. Ikot, U.S. Okorie, G. Osobonye, P.O. Amadi, C.O. Edet, M.J. Sithole, G.J. Rampho, R. Sever, Superstatistics of Schrödinger equation with pseudo-harmonic potential in external magnetic and Aharanov-Bohm fields, Heliyon 6 (2020) e03738, https://doi.org/10.1016/j.heliyon.2020.e03738.

[2] A.N. Ikot, U.S. Okorie, A.T. Ngiangia, C.A. Onate, C.O. Edet, I.O. Akpan, P.O. Amadi, Bound state solutions of the Schrödinger equation with energy dependent molecular Kratzer potential via asymptotic iteration method, Eclet. Quím. J. 45 (2020) 65, https://doi.org/10.26850/1678-4618eqj.v45.1.p65-76.

[3] H. Louis, B.I. Ita, T.O. Magu, O.U. Akakuru, N.A. Nzeata-Ibe, A.I Ikeuba, A.I. Pigweh and C.O. Edet, Solutions to the Dirac Equation for Manning-Rosen Plus Shifted Deng-Fan Potential and Coulomb-Like 
Tensor Interaction Using Nikiforov-Uvarov Method, Intl. J. Chem. 10 (2018) 99, https://doi.org/10.5539/ijc.v10n3p99.

[4] C.O. Edet, U.S. Okorie, A.T. Ngiangia and A.N. Ikot, Bound state solutions of the Schrodinger equation for the modified Kratzer potential plus screened Coulomb potential, Ind. J. Phys. 94 (2019) 425 , https://doi.org/10.1007/s12648-019-01467-x.

[5] C.O. Edet, K.O. Okorie, H. Louis and N.A. Nzeata-Ibe, Any l-state solutions of the Schrodinger equation interacting with HellmannKratzer potential model, Indian J Phys 94 (2020) 243, https://doi.org/10.1007/s12648-019-01477-9.

[6] H. Louis, B.I. Ita, O.U. Akakuru, N.A. Nzeata-Ibe, A.I. Ikeuba, T.O. Magu, P.I. Amos and C.O. Edet, $l$-state Solutions of the Relativistic and Non-Relativistic Wave Equations for Modified Hylleraas-Hulthen Potential Using the Nikiforov-Uvarov Quantum Formalism, Oriental J. Phys. Sci. 3 (2018) 1. http://www.orientjphysicalsciences.org/.

[7] G. J. Rampho, A. N. Ikot , C. O. Edet \& U. S. Okorie, Energy spectra and thermal properties of diatomic molecules in the presence of magnetic and $A B$ fields with improved Kratzer potential, Mol. Phys, (2020) https://doi.org/10.1080/00268976.2020.1821922.

[8] C O Edet, P O Okoi, A S Yusuf, P O Ushie and P O Amadi, Bound state solutions of the generalized shifted Hulthe'n potential, Indian $\mathrm{J}$ Phys. (2019) https://doi.org/10.1007/s12648-019-01650-0.

[9] A. N. Ikot, C. O. Edet, P. O. Amadi, U. S. Okorie, G. J. Rampho, and H. Y. Abdullah, Thermodynamic properties of Aharanov-Bohm (AB) and magnetic fields with screened Kratzer potential, Eur. Phys. J. D 74 (2020) 159, https://doi.org/10.1140/epjd/e2020-10084-9.

[10] C. O. Edet, P. O. Okoi, S. O. Chima, Analytic solutions of the Schrödinger equation with non-central generalized inverse quadratic Yukawa potential, Rev. Bras. Ens. Fís. 42 (2019) e20190083. https://doi.org/10.1590/1806-9126-RBEF-2019-0083.

[11] P.O. Okoi, C.O. Edet and T.O.Magu, Relativistic treatment of the Hellmann-generalized Morse potential, Rev. Mex. Fis. 66 (2020) 1, https://doi.org/10.31349/RevMexFis.66.1

[12] C.O. Edet and P.O. Okoi, Any l-state solutions of the Schrödinger equation for q-deformed Hulthen plus generalized inverse quadratic Yukawa potential in arbitrary dimensions Rev. Mex. Fis. 65 (2019) 333, https://doi.org/10.31349/RevMexFis.65.333.

[13] C.O. Edet, U.S. Okorie, G. Osobonye, A.N. Ikot, G.J. Rampho and R. Sever, Thermal properties of Deng-Fan-Eckart potential model using Poisson summation approach, J. Math. Chem. 58 (2020) 989. https://doi.org/10.1007/s10910-020-01107-4.

[14] U. S. Okorie, A. N. Ikot, C. O. Edet, I. O. Akpan, R. Sever and R. Rampho, Solutions of the Klein Gordon equation with generalized hyperbolic potential in D-dimensions, J. Phys. Commun. 3 (2019) 095015, https://doi.org/10.1088/2399-6528/ab42c6.

[15] B.I. Ita, H. Louis, O.U. Akakuru, N.A. Nzeata-Ibe, A.I. Ikeuba, T.O Magu, P.I. Amos and C.O. Edet, Approximate Solution to the Schrödinger Equation with Manning-Rosen plus a Class of Yukawa Potential via WKBJ Approximation Method, Bulg. J. Phys. 45 (2018) 323. https://www.bjp-bg.com/paper1.php?id=914.

[16] C. O. Edet, P. O. Amadi, M. C. Onyeaju, U. S. Okorie, R. Sever, G. J. Rampho, Hewa Y. Abdullah, Idris H. Salih and A. N. Ikot, Thermal Properties and Magnetic Susceptibility of Hellmann Potential in Aharonov-Bohm (AB) Flux and Magnetic Fields at Zero and Finite Temperatures. Journal of Low Temperature Physics (2020). https://doi.org/10.1007/s10909-020-02533-z.

[17] C.O. Edet, P.O. Amadi, U.S. Okorie, A. Tas, A.N. Ikot and G. Rampho, Solutions of Schrödinger equation and thermal properties of generalized trigonometric P" oschl-Teller potential. (2020). Rev. Mex. F1s. 66 (2020) 824. https://doi.org/10.31349/RevMexFis.66.824.

[18] W. Hua, Four-parameter exactly solvable potential for diatomic molecules. Phys. Rev. A, 42 (1990)2524.
[19] G. H. Sun \& S. H. Dong, Relativistic treatment of spinless particles subject to a Tietz-Wei oscillator. Communications in Theoretical Physics, 58 (2012) 195.

[20] A. N. Ikot, E. Maghsoodi, E. Ibanga, E. Ituen, \& H. Hassanabadi, Bound states of the Dirac equation for Modified Mobius square potential within the Yukawa-like tensor interaction. Proceedings of the National Academy of Sciences, India Section A: Physical Sciences, 86 (2016) 433.

[21] U S Okorie, C O Edet, A N Ikot, G J Rampho and R Sever, Thermodynamic functions for diatomic molecules with modified Kratzer plus screened Coulomb potential, Indian J Phys (2020) https://doi.org/10.1007/s12648-019-01670-w

[22] Ekpo, C. M., Inyang, E. P., Okoi, P. O, Magu, T. O., Agbo, E. P., Okorie, K. O., Inyang, E. P., (2020) New Generalized Morse-Like Potential for Studying the Atomic Interaction in Diatomic Molecules.arXiv.2012.02581[quant-ph].

[23] Yazarloo BH, Hassanabadi H, Zarrinkamar S (2012) Oscillator strengths based on the Mobius square potential under Schrödinger equation. EPJ Plus 127:51s.

[24] P. O. Amadi, C. O.Edet, U. S. Okorie, G. T. Osobonye, G. J. Rampho \& A. N. Ikot, Superstatistics of the screened Kratzer potential with Modified Dirac Delta and Uniform Distributions. (2020) arXiv preprint arXiv:2001.10496.

[25] C. O. Edet, P. O. Amadi, A. N. Ikot, U. S. Okorie, A. Tas \& G. J. Rampho (2019). Thermodynamic properties of the Superstatistics and Normal Statistics of the Schrodinger Equation with generalized trigonometric Poschl Teller potential. arXiv preprint arXiv: 1912.00148.

[26] Edet, C. (2020). Effects of Magnetic And Aharanov-Bohm (AB) Fields on the Energy Spectra of the Yukawa Potential. arXiv preprint arXiv:2012.08644.

[27] A. N. Ikot, U. S. Okorie, G. J. Rampho, P. O. Amadi, C. O. Edet, I. O. Akpan, H. Y. Abdullah \& R. Horchani, Klein-Gordon Equation and Nonrelativistic Thermodynamic Properties with Improved Screened Kratzer Potential. Journal of Low Temperature Physics, 1-21. (2021) https://doi.org/10.1007/s10909-020-02544-w.

[28] Ikot, A. N., Okorie, U. S., Amadi, P. O., Edet, C. O., Rampho, G. J., \& Sever, R. (2021). The Nikiforov-Uvarov-Functional Analysis (NUFA) Method: A New Approach for Solving Exponential-Type Potentials. Few-Body Systems, 62(1), 1-16.

[29] Edet, C. O., Ikot, A. N., Onyeaju, M. C., Okorie, U. S., Rampho, G. J. Lekala, M. L., \& Kaya, S. (2021). Thermo-Magnetic Properties of the Screened Kratzer potential with Spatially varying mass under the influence of Aharanov-Bohm (AB) and Position-Dependent Magnetic fields. Physica E: Low-dimensional Systems and Nanostructures, 114710.

[30] Ikot, A. N., Edet, C. O., Okorie, U. S., Abdel-Aty, A. H., Ramantswana, M., Rampho, G. J., \& Kaya, S. (2021). Solutions of the 2D Schrodinger equation and its thermal properties for improved ultra-generalized exponential hyperbolic potential (IUGE-HP). The European Physical Journal Plus, 136(4), 1-18

[31] Edet, C. O., \& Ikot, A. N. (2021). Effects of Topological Defect on the Energy Spectra and Thermo-magnetic Properties of \$\$ CO \$ CO Diatomic Molecule. Journal of Low Temperature Physics, 203(1), 84 111.

[32] Edet, C. O., \& Ikot, A. N. (2021). Shannon information entropy in the presence of magnetic and Aharanov-Bohm (AB) fields. The European Physical Journal Plus, 136(4), 1-11.

[33] Okorie, U. S., Ikot, A. N., Edet, C. O., Rampho, G. J., Horchani, R., \& Jelassi, H. (2021). Bound and scattering states solutions of the KleinGordon equation with generalized Mobius square potential in Ddimensions. The European Physical Journal D, 75(2), 1-8. 